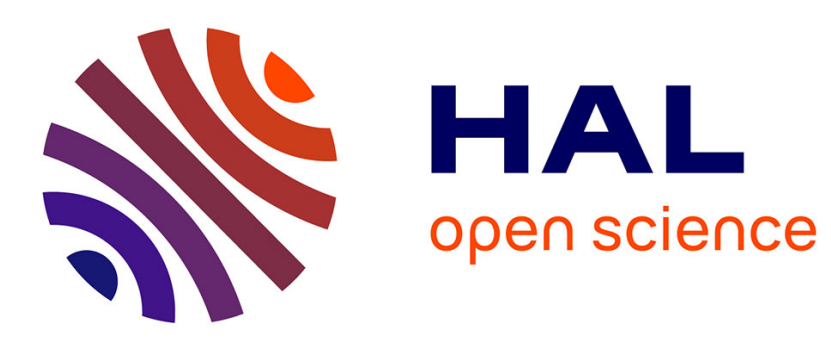

\title{
Line-shapes in Doppler-free two-photon spectroscopy. The effect of finite transit time
}

\author{
F. Biraben, M. Bassini, B. Cagnac
}

\section{To cite this version:}

F. Biraben, M. Bassini, B. Cagnac. Line-shapes in Doppler-free two-photon spectroscopy. The effect of finite transit time. Journal de Physique, 1979, 40 (5), pp.445-455. 10.1051/jphys:01979004005044500 . jpa-00209125

\section{HAL Id: jpa-00209125 https://hal.science/jpa-00209125}

Submitted on 1 Jan 1979

HAL is a multi-disciplinary open access archive for the deposit and dissemination of scientific research documents, whether they are published or not. The documents may come from teaching and research institutions in France or abroad, or from public or private research centers.
L'archive ouverte pluridisciplinaire HAL, est destinée au dépôt et à la diffusion de documents scientifiques de niveau recherche, publiés ou non, émanant des établissements d'enseignement et de recherche français ou étrangers, des laboratoires publics ou privés. 


\title{
Line-shapes in Doppler-free two-photon spectroscopy. The effect of finite transit time
}

\author{
F. Biraben, M. Bassini and B. Cagnac \\ Laboratoire de Spectroscopie Hertzienne de l'E.N.S., Université P.-et-M.-Curie, place Jussieu, 75005 Paris Cedex 05, France
}

(Reçu le 8 décembre 1978, accepté le 24 janvier 1979)

\begin{abstract}
Résumé. - On développe un formalisme général permettant de calculer la forme des raies d'absorptịon à deux photons dans le cas de champs non monochromatiques. Ce formalisme est appliqué au problème du temps de transit fini des atomes à travers le faisceau Laser, et permet d'obtenir une expression précise des formes de raies (convolution d'une Lorentzienne et d'une double exponentielle). La comparaison est faite avec des profils de raies expérimentaux.
\end{abstract}

\begin{abstract}
A general formalism is developed with the aim of calculating the line-shapes in two-photon absorption for the case of non monochromatic fields. This formalism is applied to the problem of the finite transit time of the atoms through the Laser beam ; and it permits a precise expression to be obtained for the line-shape (convolution of a Lorentzian curve and a double-exponential curve). The comparison is made using experimental profiles.
\end{abstract}

Since the first experiments in 1974 [1, 2, 3], Dopplerfree two-photon spectroscopy has found a wide range of applications, a review of which can be found for instance in [4]. With the improvement of c.w. lasers has come increased accuracy, allowing detailed explanations of experimental line-shapes. The aim in this paper is to present the detailed calculation of lineshapes, emphasising in particular the role of the transit time of the atoms through the laser beam, and comparing the results with experiment. The problem of transit time has been thoroughly studied in the case of saturated absorption spectroscopy $[5,6$, $7,8]$. But the problems involved in two-photon absorption are quite different and more simple, because the part of the Hamiltonian corresponding to the Doppler free line in a stationary field is independent of the atomic velocity.

In the first section we deal with the interaction of an atom with a monochromatic light field in the steady state. The results have already been published in references [9] and [10], but we introduce our notations and justify the preliminary formulas which will be used in the following sections.

In the second section we develop a general formalism, suitable for the case of non-monochromatic fields, with which one can easily include the effects of finite transit times and of collisions.

In the third section we apply these general formulas to the transit time problem. The calculated line-shape is found to be in agreement with that calculated by Bordé [11] in the case of a three-level system.

In the fourth and final section we present experimental spectral profiles and demonstrate that these are well interpreted with our calculations.

1. Case of monochromatic field in the steady state. Consider the process in which an atom absorbs two photons, each of energy $\hbar \omega$, from a laser beam and thereby undergoes a transition from the ground state $|\mathrm{g}\rangle$ to an excited state $|\mathrm{e}\rangle$ across an energy interval $E_{\mathrm{e}}-E_{\mathrm{g}}=\hbar \omega_{\mathrm{ge}}$ (see Fig. 1). We suppose that this process is predominant, with a small energy detuning :

$$
\delta \omega=\omega-\frac{\omega_{\mathrm{ge}}}{2}
$$

from the resonant, two-step transition frequency. Other single photon transitions between $|\mathrm{g}\rangle$ and other excited levels $|\mathrm{r}\rangle$, of energy $E_{\mathrm{r}}=E_{\mathrm{g}}+\hbar \omega_{\mathrm{gr}}$, are assumed to be far from resonance, i.e. the energy defect

$$
\Delta \omega_{\mathrm{r}}=\omega-\omega_{\mathrm{gr}}
$$

is taken to be large.

The aim in this section is to recall the fundamental formulas needed as a basis for our calculations, and in particular to introduce the two-photon operator. 


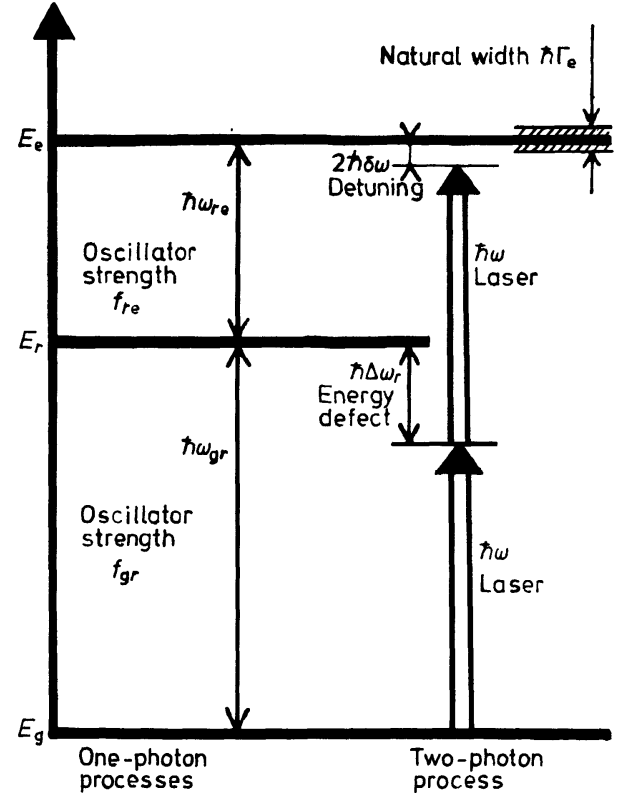

Fig. 1. - Energy diagram of a two-photon transition.

This operator has been introduced in reference [10] (see also [12, 13, 14]) using the dressed-atom formalism in the atomic rest-frame. Here it is more convenient to use time dependent perturbation theory in the laboratory frame.

Nevertheless we include in the Hamiltonian $\mathscr{H}_{1}$ of the free atom the relaxation due to spontaneous emission. It is indeed possible to describe the coupling of the excited state with the vacuum modes of the electromagnetic field by addition of an imaginary part to the Hamiltonian $\mathscr{H}_{0}$ of the free atom [15].

The atomic Hamiltonian is then

$$
\mathscr{H}_{1}=\mathscr{H}_{0}-i \hbar \Gamma / 2,
$$

$\mathscr{H}_{0}$ is the Hamiltonian in the absence of spontaneous emission and $\Gamma$ is an operator acting only on the excited states $|\mathrm{e}\rangle$ and $|\mathrm{r}\rangle$, such that for any state $\alpha$ of radiative decay rate $\Gamma_{\alpha}$

$$
\mathscr{H}_{1}|\alpha\rangle=\hbar\left(\omega_{\mathrm{g} \alpha}-i \frac{\Gamma_{\alpha}}{2}\right)|\alpha\rangle \text {. }
$$

Here we assume the conventional value zero for the energy of the ground state $|g\rangle$. Such a non Hermitian Hamiltonian must be used with great caution. But we have no problems in our case, as we remain in the frame of perturbation theory and we suppose that the two-photon transition is far from saturation. The Hamiltonian of the atom interacting with the electromagnetic field of the laser is $H_{1}+V$, where $V$ represents the interaction between the radiation field $\mathbf{E}$ and the atom. In the electric dipole approximation this term can be written :

$$
V=-\mathbf{D} \cdot \mathbf{E},
$$

$\mathbf{D}$ is the dipole moment operator of the atom.

The atoms are submitted to a stationary field, obtained by the superposition of two electric fields with the same frequency, and which can be written in the laboratory frame :

$$
\begin{aligned}
& \mathbf{E}_{1}(\mathbf{r}, t)=\varepsilon_{1} E_{1} \mathrm{e}^{i\left(\mathbf{k}_{1} \cdot \mathbf{r}-\omega t\right)}+\varepsilon_{1}^{*} E_{1}^{*} \mathrm{e}^{-i\left(\mathbf{k}_{1} \cdot \mathbf{r}-\omega t\right)} \\
& \mathbf{E}_{2}(\mathbf{r}, t)=\varepsilon_{2} E_{2} \mathrm{e}^{i\left(\mathbf{k}_{2} \cdot \mathbf{r}-\omega t\right)}+\varepsilon_{2}^{*} E_{2}^{*} \mathrm{e}^{-i\left(\mathbf{k}_{2} \cdot \mathbf{r}-\omega t\right)}
\end{aligned}
$$

following the convention of Heitler [16], the first term (with $\mathrm{e}^{-i \omega t}$ ) being effective for absorption, whereas the second complex conjugate term being effective for emission. $\varepsilon_{i}$ are the polarization vectors, $E_{i}$ the amplitudes, $\mathbf{k}_{i}$ the wave vectors $(i=1,2)$. We restrict the calculation to the condition of Doppler free experiments where $\mathbf{k}_{1}+\mathbf{k}_{2}=0$ (the laser beam is reflected back on itself by a mirror). Let us set :

$$
D_{\varepsilon_{i}}=\mathbf{D} . \varepsilon_{i} \text {. }
$$

Supposing that the atom first absorbs a photon from wave 1 and then a photon from wave 2 , the vector state at time $t$ of an atom initially in the ground state can be calculated using second order, time-dependent perturbation theory :

$$
|\Psi(t)\rangle=-\frac{1}{\hbar^{2}} \int_{-\infty}^{t} \mathrm{~d} t_{2} \int_{-\infty}^{t_{2}} \mathrm{~d} t_{1} E_{1} E_{2} \mathrm{e}^{-i \omega\left(t_{2}+t_{1}\right)} \times \mathrm{e}^{i\left[\mathbf{k}_{2} \cdot \mathbf{r}\left(t_{2}\right)+\mathbf{k}_{1} \cdot \mathbf{r}\left(t_{1}\right)\right]} \mathrm{e}^{-i \mathscr{S}_{1}\left(t-t_{2}\right) / \hbar} D_{\varepsilon_{2}} \mathrm{e}^{-i \mathcal{S}_{1}\left(t_{2}-t_{1}\right) / \hbar} D_{\varepsilon_{1}}|\mathrm{~g}\rangle .
$$

Setting $u=t_{2}-t_{1}$ and using the condition $\mathbf{k}_{2}=-\mathbf{k}_{1}$ we obtain

$$
|\Psi(t)\rangle=-\frac{1}{\hbar^{2}} \int_{-\infty}^{t} \mathrm{~d} t_{2} \int_{0}^{+\infty} \mathrm{d} u E_{1} E_{2} \mathrm{e}^{-2 i \omega t_{2}} \mathrm{e}^{-i \mathscr{K}_{1}\left(t-t_{2}\right) / \hbar} D_{\varepsilon_{2}} \mathrm{e}^{i\left(\omega-\mathbf{k}_{1} \cdot \mathrm{v}-\mathfrak{K}_{1} / \hbar\right) u} D_{\varepsilon_{1}}|\mathrm{~g}\rangle .
$$

It is important to notice that the velocity $\mathbf{v}$ remains only in the coefficient of $u$, not in the coefficient of $t_{2}$. Performing the integration on $u$ we obtain :

$$
|\Psi(t)\rangle=\frac{i}{\hbar} \int_{-\infty}^{t} \mathrm{~d} t_{2} E_{1} E_{2} \mathrm{e}^{-2 i \omega t_{2}} \mathrm{e}^{-i \mathscr{H}_{1}\left(t-t_{2}\right) / \hbar} D_{\varepsilon_{2}} \frac{1}{\mathcal{H}_{1}-\hbar\left(\omega-\mathbf{k}_{1} \cdot \mathbf{v}\right)} D_{\varepsilon_{1}}|\mathrm{~g}\rangle .
$$


As we have supposed that all levels $|r\rangle$ were far off resonance for one-photon transition from the ground state, we can neglect the relaxation rate $\Gamma_{\mathrm{r}}$ and the Doppler shift $\mathbf{k}_{1} \cdot \mathbf{v}$ in comparison with the energy defect in the intermediate levels :

$$
\left|\Delta \omega_{\mathrm{r}}\right|=\left|\omega-\omega_{\mathrm{gr}}\right| \gg \Gamma_{\mathrm{r}} \text { and } \mathbf{k}_{1} \cdot \mathbf{v}
$$

and we can make the approximation :

$D_{\varepsilon_{2}} \frac{1}{H_{1}-\hbar\left(\omega-\mathbf{k}_{1} \cdot \mathbf{v}\right)} D_{\varepsilon_{1}} \simeq D_{\varepsilon_{2}} \frac{1}{H_{0}-\hbar \omega} D_{\varepsilon_{1}}=Q_{\varepsilon_{1} \varepsilon_{2}}$

$Q_{\varepsilon_{1} \varepsilon_{2}}$ is the two-photon transition operator.

We must then add to (10) the contribution from the process in which the atom absorbs a photon of wave 2 first, followed by a photon of wave 1 . The expression for the state vector $\Psi(t)$ will contain the symmetrical two-photon transition operator $Q_{\varepsilon_{1} \varepsilon_{2}}^{\mathrm{S}}$

$$
Q_{\varepsilon_{1} \varepsilon_{2}}^{\mathrm{S}}=\frac{1}{2}\left(Q_{\varepsilon_{1} \varepsilon_{2}}+Q_{\varepsilon_{2} \varepsilon_{1}}\right) \text {. }
$$

As a consequence of the condition (11), the atomic velocity $\mathbf{v}$ has disappeared from the expression (10) and the calculated result will be independent of velocity, i.e. without Doppler broadening.

We must now perform the remaining integration of (10), project to the final state $|\mathrm{e}\rangle$ and hence deduce the transition probability from $g$ to e per unit time $\Gamma_{\mathrm{ge}}^{(2)}$, which is produced by absorbing one photon from each travelling wave :

$$
\Gamma_{\mathrm{ge}}^{(2)}=\frac{4 E_{1}^{2} E_{2}^{2}}{\hbar^{2}}\left|\left\langle\mathrm{e}\left|Q_{\varepsilon_{1} \varepsilon_{2}}^{\mathrm{s}}\right| \mathrm{g}\right\rangle\right|^{2} \frac{\Gamma_{\mathrm{e}}}{4 \delta \omega^{2}+\Gamma_{\mathrm{e}}^{2} / 4}
$$

It is still necessary to include the contribution from the processes in which two photons of the same travelling wave are absorbed. These additional terms are velocity dependent. One obtains the complete formula

$$
\begin{aligned}
\Gamma_{\mathrm{ge}}^{(2)}=\frac{4 E_{1}^{2} E_{2}^{2}}{\hbar^{2}}\left|\left\langle\mathrm{e}\left|Q_{\varepsilon_{1} \varepsilon_{2}}^{\mathrm{s}}\right| \mathrm{g}\right\rangle\right|^{2} \frac{\Gamma_{\mathrm{e}}}{4 \delta \omega^{2}+\Gamma_{\mathrm{e}}^{2} / 4} & +\frac{E_{1}^{4}}{\hbar^{2}}\left|\left\langle\mathrm{e}\left|Q_{\varepsilon_{1} \varepsilon_{1}}\right| \mathrm{g}\right\rangle\right|^{2} \frac{\Gamma_{\mathrm{e}}}{4\left(\delta \omega-k v_{\mathrm{z}}\right)^{2}+\Gamma_{\mathrm{e}}^{2} / 4} \\
& +\frac{E_{2}^{4}}{\hbar^{2}}\left|\left\langle\mathrm{e}\left|Q_{\varepsilon_{2} \varepsilon_{2}}\right| \mathrm{g}\right\rangle\right|^{2} \frac{\Gamma_{\mathrm{e}}}{4\left(\delta \omega+k v_{\mathrm{z}}\right)^{2}+\Gamma_{\mathrm{e}}^{2} / 4}
\end{aligned}
$$

where $k$ is the common modulus of the wave vectors $\mathbf{k}_{1}$ and $\mathbf{k}_{2}$ and $v_{z}$ the component of the atomic velocity $\mathbf{v}$ in the laser beam direction. To obtain the absorption line shape as a function of the angular frequency $\omega$ of the laser light, we have to average (15) over the velocity distribution $f\left(v_{z}\right)$ of the atoms

$$
f\left(v_{z}\right)=\frac{1}{u \sqrt{\pi}} \mathrm{e}^{-v_{z}^{2} / u^{2}} \quad \text { where } \quad u^{2}=\frac{2 k T}{M}
$$

( $k$ Boltzmann constant, $M$ atomic mass).

Averaging the three terms of (15), one obtains a broad Gaussian curve, of Doppler width

$$
\Delta \omega_{\mathrm{D}}=2 k u \log 2,
$$

from the last two terms, together with a narrow Lorentzian curve, of width $\Delta \omega_{\mathrm{N}}=\Gamma_{\mathrm{e}} / 2$, from the first term (the latter width is half the natural width of the excited level because we have calculated the absorption as a function of the frequency detuning $\delta \omega$ for one exciting photon). When the polarizations $\varepsilon_{1}$ and $\varepsilon_{2}$ of the two waves are identical, one can see from (15) that the probability of absorbing two photons propagating in opposite directions is four times the probability of absorbing two photons from the same wave : the area under the Lorentzian curve is twice that under the Gaussian [10].

Owing to the fact that the Doppler width is usually 100 or 1000 times larger than the natural width and the areas of the two curves are of the same order of magnitude, it follows that the height of the narrow Lorentzian curve is 100 or 1000 times greater than that of the Gaussian; the latter curve appearing as a very small background.

As an example, we reproduce in figure 2 an experimental recording of the amount of two-photon absorption as a function of the laser frequency for the transition $3 S \rightarrow 4 D$ in sodium [17]. In this recording, the relative importance of the Gaussian background is increased owing to the presence of four

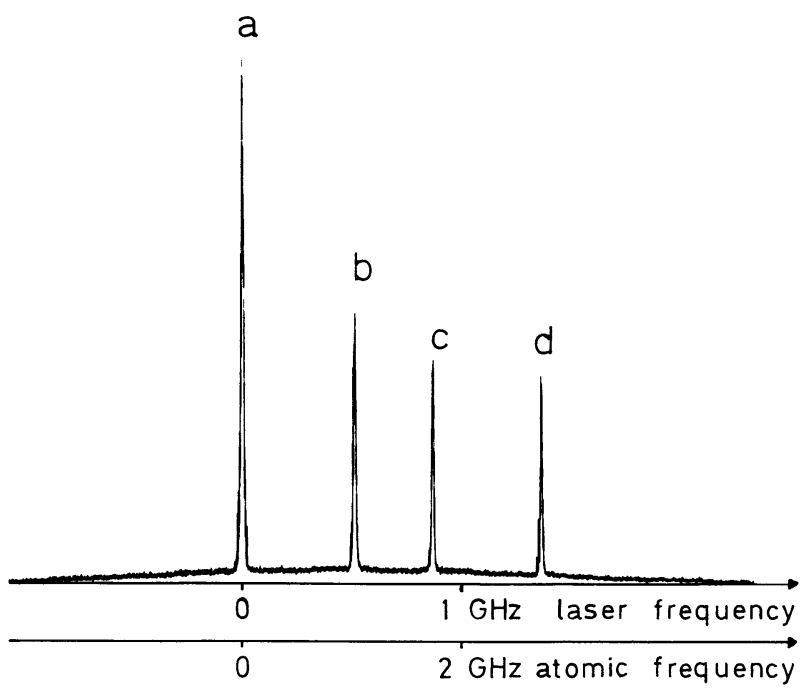

Fig. 2. - Experimental recording of a two-photon absorption line $3 S \rightarrow 4 D$ in sodium (Number of photons reemitted from the final excited state versus the Laser frequency). 
distinct transitions within the Doppler width, the corresponding Gaussian curves being superimposed.

In high resolution experiments it is necessary to take into account the shape of this Gaussian background.

For our purpose, however, we need only consider the absorption of two oppositely-travelling photons - this principally determining the line-shape of the narrow peaks - and we thus neglect the broad background.

Formula (14) can also be used to evaluate the absolute value of the transition probability as a function of the light beam intensity, that is, of the power per unit area

$$
\frac{P_{i}}{S_{i}}=2 \varepsilon_{0} c E_{i}^{2}
$$

(not forgetting, however, that the amplitudes of the classical electric fields corresponding to expression (6) are $2 E_{i}$ ). Examples of such calculations can be found in references [10] and [4]. Here we will be interested only in relative values of the transition probabilities at various laser frequencies.

2. General formalism for the case of non-monochromatic fields. - We now generalize the calculations of section 1. To facilitate this generalization, we consider here the electric field $E(t)$ experienced by the atom in its own frame, while the relation between $E(t)$ and the field of the laser beam in the laboratory frame will be considered in section 3. $E(t)$ is determined by its Fourier decomposition

$\mathbf{E}(t)=\varepsilon \int_{0}^{\infty} \mathrm{d} \omega E(\omega) \mathrm{e}^{-i \omega t}+\varepsilon^{*} \int_{0}^{\infty} \mathrm{d} \omega E^{*}(\omega) \mathrm{e}^{+i \omega t}$

We choose this form of decomposition (with $\omega$ positive) in order to distinguish the terms in $\mathrm{e}^{-i \omega t}$, which are effective in absorption, from those in $\mathrm{e}^{+i \omega t}$ which are effective in emission. We assume that the function $E(\omega)$ is centred at a value $\omega_{\mathrm{L}}$ corresponding to a small energy detuning (eq. (1)), and that the function's width, $\Delta \omega_{L}$, is sufficiently narrow to neglect one-photon transitions, i.e.

$$
\left|\Delta \omega_{\mathbf{r}}\right|=\left|\omega-\omega_{\mathbf{g r}}\right| \stackrel{\sim}{\gg} \Delta \omega_{\mathbf{L}} .
$$

The energy absorbed by one atom while in the laser field may be obtained by calculating the work done, $W$, by the electric field $E(t)$ acting on the induced atomic dipole :

$$
W=\int_{T_{1}}^{T_{2}} \mathrm{~d} t \mathbf{E}(t) \cdot \frac{\mathrm{d}}{\mathrm{d} t}\langle\Psi(t)|\mathbf{D}| \Psi(t)\rangle .
$$

We perform this calculation under the hypothesis that the electric field $E(t)$ is zero before $T_{1}$ and after $T_{2}$ :

$$
E(t)=0 \text { for }\left\{\begin{array}{l}
t<T_{1} \\
t>T_{2}
\end{array}\right.
$$

$\Delta T=T_{2}-T_{1}$, may be a pulse duration or the transit time of the atom through the laser field.

Under this hypothesis, it is possible to extend the limits of integration in (19) to infinity. (We will see later that a generalization is possible in the case of stationary fields.) Using the components, $D_{\varepsilon}$, of the dipole moment operator defined by (7) (and noting that $D_{\varepsilon}^{*}=D_{\varepsilon}^{+}$), we find that the absorbed energy can be written :

$$
\begin{aligned}
W=2 \operatorname{Re} \int_{-\infty}^{+\infty} & \mathrm{d} t \int_{0}^{\infty} \mathrm{d} \omega E^{*}(\omega) \times \\
& \times \mathrm{e}^{+i \omega t} \frac{\mathrm{d}}{\mathrm{d} t}\left\langle\Psi(t)\left|D_{\varepsilon}^{+}\right| \Psi(t)\right\rangle .
\end{aligned}
$$

Among the various terms obtained in the exact calculation of the mean value $\left\langle D_{\varepsilon}^{+}\right\rangle$, only those in $\mathrm{e}^{-i \omega t}$ will give important contributions to the integral (21), and these terms only will be retained in the following.

In order to calculate the vector state $|\Psi(t)\rangle$ needed in the evaluation of $\left\langle D_{\varepsilon}^{+}\right\rangle$, we use time-dependent perturbation theory with the perturbation Hamiltonian given by (5). We choose to perform the calculations in the Schrödinger representation, and not in the interaction representation, since this allows us to use the unperturbed Hamiltonian $\mathcal{H}_{1}$ (eq. (3)) which is non-Hermitian, thereby including the effects of relaxation by spontaneous emission. In order to simplify the formulas we introduce the notation :

$$
H_{1}=\frac{\mathscr{H}_{1}}{\hbar}=\frac{\mathscr{H}_{0}}{\hbar}-i \frac{\Gamma}{2} \text {. }
$$

As we will see, it is necessary to develop the perturbation calculation to third order. However, we will retain only those terms corresponding to the absorption of two photons (thus neglecting induced emission processes by assuming that the two-photon transition is far below saturation). To first and second order, the retained terms are those from the term in $\mathrm{e}^{-i \omega t}$ in the electric field expression (17); the third order term is more complex :

$$
\begin{aligned}
|\Psi(t)\rangle=|\mathrm{g}\rangle & +\frac{i}{\hbar} \int_{-\infty}^{t} \mathrm{~d} t_{1} \int_{0}^{\infty} \mathrm{d} \omega_{1} E\left(\omega_{1}\right) \mathrm{e}^{-i \omega_{1} t_{1}} \mathrm{e}^{-i H_{1}\left(t-t_{1}\right)} D_{\varepsilon}|\mathrm{g}\rangle \\
& -\frac{1}{\hbar^{2}} \int_{-\infty}^{t} \mathrm{~d} t_{2} \int_{-\infty}^{t_{2}} \mathrm{~d} t_{3} \iint_{0}^{\infty} \mathrm{d} \omega_{2} \mathrm{~d} \omega_{3} E\left(\omega_{2}\right) E\left(\omega_{3}\right) \mathrm{e}^{-i \omega_{2} t_{2}} \mathrm{e}^{-i \omega_{3} t_{3}} \mathrm{e}^{-i H_{1}\left(t-t_{2}\right)} D_{\varepsilon} \mathrm{e}^{-i H_{1}\left(t_{2}-t_{3}\right)} D_{\varepsilon}|\mathrm{g}\rangle \\
& -\frac{i}{\hbar^{3}} \int_{-\infty}^{t} \mathrm{~d} t_{1} \int_{-\infty}^{t_{1}} \mathrm{~d} t_{2} \int_{-\infty}^{t_{2}} \mathrm{~d} t_{3} \iiint \mathrm{d} \omega_{1} \mathrm{~d} \omega_{2} \mathrm{~d} \omega_{3} E^{*}\left(\omega_{1}\right) E\left(\omega_{2}\right) E\left(\omega_{3}\right) \\
& \times \mathrm{e}^{+i \omega_{1} t_{1}} \mathrm{e}^{-i \omega_{2} t_{2}} \mathrm{e}^{-i \omega_{3} t_{3}} \mathrm{e}^{-i H_{1}\left(t-t_{1}\right)} D_{\varepsilon}^{+} \mathrm{e}^{-i H_{1}\left(t_{1}-t_{2}\right)} D_{\varepsilon} \mathrm{e}^{-i H_{1}\left(t_{2}-t_{3}\right)} D_{\varepsilon}|\mathrm{g}\rangle .
\end{aligned}
$$


Performing the integrals over $t_{3}$ in the second order and third order terms is analogous to transforming equation (9) into (10) : the relaxation time inside the Hamiltonian $H_{1}$ reduces to zero the term corresponding to one of the two time-limits of integration.

Further, the conditions (11) and (18) allow the approximation necessary for the introduction of the twophoton operator to be made (12). Introducing the new variables $\theta=t-t_{1}$ and $\tau=t-t_{2}$ or $\tau=t_{1}-t_{2}$, we obtain

$$
\begin{aligned}
|\Psi(t)\rangle=|\mathrm{g}\rangle & +\frac{i}{\hbar} \int_{0}^{\infty} \mathrm{d} \omega_{1} E\left(\omega_{1}\right) \mathrm{e}^{-i \omega_{1} t} \int_{0}^{+\infty} \mathrm{d} \theta \mathrm{e}^{+i \omega_{1} \theta} \mathrm{e}^{-i H_{1} \theta} D_{\varepsilon}|\mathrm{g}\rangle \\
& +\frac{i}{\hbar} \iint_{0}^{\infty} \mathrm{d} \omega_{2} \mathrm{~d} \omega_{3} E\left(\omega_{2}\right) E\left(\omega_{3}\right) \mathrm{e}^{-i\left(\omega_{2}+\omega_{3}\right) t} \int_{0}^{+\infty} \mathrm{d} \tau \mathrm{e}^{i\left(\omega_{2}+\omega_{3}\right) \tau} \mathrm{e}^{-i H_{1} \tau} Q_{\varepsilon \varepsilon}|\mathrm{g}\rangle \\
& -\frac{1}{\hbar^{2}} \iiint_{0}^{\infty} \mathrm{d} \omega_{1} \mathrm{~d} \omega_{2} \mathrm{~d} \omega_{3} E^{*}\left(\omega_{1}\right) E\left(\omega_{2}\right) E\left(\omega_{3}\right) \mathrm{e}^{-i\left(\omega_{2}+\omega_{3}-\omega_{1}\right) t} \\
& \times \int_{0}^{+\infty} \mathrm{d} \theta \int_{0}^{+\infty} \mathrm{d} \tau \mathrm{e}^{i\left(\omega_{2}+\omega_{3}-\omega_{1}\right) \theta} \mathrm{e}^{-i H_{1} \theta} D_{\varepsilon}^{+} \mathrm{e}^{i\left(\omega_{2}+\omega_{3}\right) \tau} \mathrm{e}^{-i H_{1} \tau} Q_{\varepsilon \varepsilon}|\mathrm{g}\rangle .
\end{aligned}
$$

In calculating the mean value $\left\langle\Psi\left|D_{\varepsilon}^{+}\right| \Psi\right\rangle$, we exclude those terms which correspond to one photon transitions (see hypothesis (1) and (2)) and we retain only those which contribute to the integral (21). In fact there are only two such terms : one is obtained from the product of first-order and second-order terms in (24), while the other is got from a product of zero-order and third-order terms. These two terms correspond to the absorption of one photon in each of the two steps of the complete process $(\mathrm{g} \rightarrow \mathrm{r}$ and $\mathrm{r} \rightarrow \mathrm{e})$ :

$$
\begin{aligned}
\left\langle\Psi(t)\left|D_{\varepsilon}^{+}\right| \Psi(t)\right\rangle= & +\frac{1}{\hbar^{2}} \iiint \mathrm{d} \omega_{1} \mathrm{~d} \omega_{2} \mathrm{~d} \omega_{3} E^{*}\left(\omega_{1}\right) E\left(\omega_{2}\right) E\left(\omega_{3}\right) \mathrm{e}^{-i\left(\omega_{2}+\omega_{3}-\omega_{1}\right) t} \\
& \times \int_{0}^{\infty} \mathrm{d} \theta \int_{0}^{\infty} \mathrm{d} \tau\left\langle\mathrm{g}\left|D_{\varepsilon}^{+} \mathrm{e}^{i\left(H_{1}^{+}-\omega_{1}\right) \theta} D_{\varepsilon}^{+} \mathrm{e}^{i\left(\omega_{2}+\omega_{3}-H_{1}\right) \tau} Q_{\varepsilon \varepsilon}\right| \mathrm{g}\right\rangle \\
& -\frac{1}{\hbar^{2}} \iiint \mathrm{d} \omega_{1} \mathrm{~d} \omega_{2} \mathrm{~d} \omega_{3} E^{*}\left(\omega_{1}\right) E\left(\omega_{2}\right) E\left(\omega_{3}\right) \mathrm{e}^{-i\left(\omega_{2}+\omega_{3}-\omega_{1}\right) t} \\
& \times \int_{0}^{\infty} \mathrm{d} \theta \int_{0}^{\infty} \mathrm{d} \tau\left\langle\mathrm{g}\left|D_{\varepsilon}^{+} \mathrm{e}^{i\left(\omega_{2}+\omega_{3}-\omega_{1}-H_{1}\right) \theta} D_{\varepsilon}^{+} \mathrm{e}^{i\left(\omega_{2}+\omega_{3}-H_{1}\right) \tau} Q_{\varepsilon \varepsilon}\right| \mathrm{g}\right\rangle .
\end{aligned}
$$

Performing the integrals over $\theta$ allows the introduction of the two-photon operator again and also shows that both terms on the right hand side of equation (25) are equal with the same sign :

$$
\begin{aligned}
\left\langle\Psi(t)\left|D_{\varepsilon}^{+}\right| \Psi(t)\right\rangle=\frac{2 i}{\hbar} \iiint \mathrm{d} \omega_{1} \mathrm{~d} \omega_{2} \mathrm{~d} \omega_{3} E^{*}\left(\omega_{1}\right) E\left(\omega_{2}\right) E\left(\omega_{3}\right) \mathrm{e}^{-i\left(\omega_{2}+\omega_{3}-\omega_{1}\right) t} \times \\
\times \int_{0}^{\infty} \mathrm{d} \tau\left\langle\mathrm{g}\left|Q_{\varepsilon \varepsilon}^{+} \mathrm{e}^{i\left(\omega_{2}+\omega_{3}-H_{1}\right) \tau} Q_{\varepsilon \varepsilon}\right| \mathrm{g}\right\rangle
\end{aligned}
$$

It is now easy to evaluate (26) and hence find the following expression for the absorbed energy (21) :

$$
\begin{aligned}
W=\frac{4}{\hbar} \operatorname{Re} \int_{-\infty}^{+\infty} \mathrm{d} t \iiint \int \mathrm{d} \omega \mathrm{d} \omega_{1} \mathrm{~d} \omega_{2} \mathrm{~d} \omega_{3} E^{*}(\omega) E^{*}\left(\omega_{1}\right) E\left(\omega_{2}\right) E\left(\omega_{3}\right) \mathrm{e}^{i\left(\omega+\omega_{1}-\omega_{2}-\omega_{3}\right) t} \times \\
\times\left(\omega_{2}+\omega_{3}-\omega_{1}\right) \int_{0}^{\infty} \mathrm{d} \tau \mathrm{e}^{i\left(\omega_{2}+\omega_{3}\right) \tau}\left\langle\mathrm{g}\left|Q_{\varepsilon \varepsilon}^{+} \mathrm{e}^{-i H_{1} \tau} Q_{\varepsilon \varepsilon}\right| \mathrm{g}\right\rangle
\end{aligned}
$$

To proceed further we make the following observations : the assumption (18) regarding the function $E(\omega)$ allows us to make the approximation

$$
\left(\omega_{2}+\omega_{3}-\omega_{1}\right) \simeq \omega_{\mathrm{L}}
$$


Further, the integral over time $t$

$$
\int_{-\infty}^{+\infty} \mathrm{d} t \mathrm{e}^{i\left(\omega+\omega_{1}-\omega_{2}-\omega_{3}\right) t}=2 \pi \delta\left(\omega+\omega_{1}-\omega_{2}-\omega_{3}\right)=2 \pi \delta\left(\Omega-\Omega^{\prime}\right)
$$

where we introduce the variables $\Omega=\omega+\omega_{1}$ and $\Omega^{\prime}=\omega_{2}+\omega_{3}$. Lastly, we define the quantity

$$
B(\Omega)=\int_{0}^{\infty} \mathrm{d} \omega E(\omega) E(\Omega-\omega)
$$

Formula (27) then takes the form :

$$
W=\frac{8 \pi \omega_{\mathrm{L}}}{\hbar} \operatorname{Re} \int_{0}^{\infty} \mathrm{d} \Omega B^{*}(\Omega) B(\Omega) \int_{0}^{\infty} \mathrm{d} \tau \mathrm{e}^{+i \Omega \tau}\left\langle\mathrm{g}\left|Q_{\varepsilon \varepsilon}^{+} \mathrm{e}^{-i H_{1} \tau} Q_{\varepsilon \varepsilon}\right| \mathrm{g}\right\rangle .
$$

This result may be expressed in a convenient form by using two-photon operator $\tilde{Q_{\varepsilon \varepsilon}}(t)$ defined in the interaction representation and related to the corresponding operator in the Schrödinger representation by the transformation

We obtain :

$$
\tilde{Q_{\varepsilon \varepsilon}}(t)=\mathrm{e}^{+i H_{1}^{+} t} Q_{\varepsilon \varepsilon} \mathrm{e}^{-i H_{1} t}
$$

$$
W=\frac{8 \pi \omega_{\mathrm{L}}}{\hbar} \operatorname{Re} \int_{0}^{\infty} \mathrm{d} \Omega|B(\Omega)|^{2} \int_{0}^{\infty} \mathrm{d} \tau \mathrm{e}^{+i \Omega \tau}\left\langle\mathrm{g}\left|{\tilde{Q_{\varepsilon \varepsilon}}}^{+}(\tau) \tilde{Q_{\varepsilon \varepsilon}}(0)\right| \mathrm{g}\right\rangle
$$

In the general case of complex i.e. circular or elliptical polarization of the light, it is necessary to use the adjoint operators $D_{\varepsilon}^{+}$or $Q_{\varepsilon \varepsilon}^{+}$in the above formulas. However, in the particular case of linear, i.e. real polarization, the formulas can be simplified. We may, in this case, write the electric field given by (17) as :

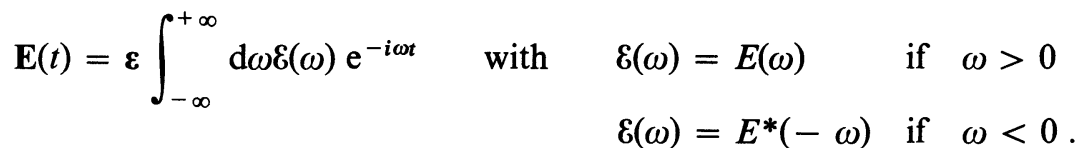

Introducing the Fourier transform $A(\Omega)$ of the square of the electric field,

$$
A(\Omega)=\frac{1}{2 \pi} \int_{-\infty}^{+\infty} \mathrm{d} t \mathrm{e}^{+i \Omega t} E(t)^{2}=\int_{-\infty}^{+\infty} \mathrm{d} \omega \mathcal{E}(\omega) \mathcal{E}(\Omega-\omega)
$$

and noting that, in the region of interest, in the vicinity of $\Omega=2 \omega_{\mathrm{L}}, A(\Omega)$ is equal to $B(\Omega)$ (as defined by (30)), then we may write the absorbed energy as :

$$
W=\frac{8 \pi \omega_{\mathrm{L}}}{\hbar} \int_{-\infty}^{+\infty} \mathrm{d} \Omega|A(\Omega)|^{2} \int_{0}^{\infty} \mathrm{d} \tau \mathrm{e}^{+i \Omega \tau}\left\langle\mathrm{g}\left|\tilde{Q_{\varepsilon \varepsilon}}(\tau) \tilde{Q_{\varepsilon \varepsilon}}(0)\right| \mathrm{g}\right\rangle .
$$

This formula is quite similar to that obtained for one-photon transitions : the Fourier transform, $A(\Omega)$, of the squared electric field replaces the Fourier transform $\&(\omega)$ of the electric field, and the correlation function of the two-photon operator $\tilde{Q_{\varepsilon \varepsilon}}$ replaces the correlation function of the dipole moment operator [18]. Note, however, that the $\tilde{Q}$ operators are defined by a non-unitary transformation, given by equations (32) and (22). Formula (36) has been obtained under the hypothesis (20) that the electric field $E(t)$ is zero before $T_{1}$ and after $T_{2}$. In order to extend the treatment to include steady-state illumination we isolate the effective interaction time of the atom with the field to an interval $T_{2}-T_{1}$ such that

$$
T_{2}-T_{1} \gg 1 / \Gamma_{\mathrm{e}} \text { and } T_{2}-T_{1} \gg 1 / \Delta \omega_{\mathrm{L}}
$$

i.e. over a finite interval long compared with characteristic evolution times of the atom. The first condition ensures the validity of the time-integrals in the perturbation calculation; the second condition, the validity of the time-integral in the expression (21) of the absorbed energy. The Fourier component $E(\omega)$ used in the formulas is then the Fourier transform of the electric field limited to the finite time 
interval between $T_{1}$ and $T_{2}$. When the electromagnetic field is the only perturbation applied to the atom, i.e. in the absence of collisions, the calculation of the correlation function is simple and the line-shape is given by

$$
\begin{aligned}
W=\frac{4 \pi \omega_{\mathrm{L}}}{\hbar}\left|\left\langle\mathrm{g}\left|Q_{\varepsilon \varepsilon}\right| \mathrm{e}\right\rangle\right|^{2} \times \\
\quad \times \int_{-\infty}^{+\infty} \mathrm{d} \Omega|A(\Omega)|^{2} \frac{\Gamma_{\mathrm{e}}}{\left(\Omega-\omega_{\mathrm{ge}}\right)^{2}+\Gamma_{\mathrm{e}}^{2} / 4}
\end{aligned}
$$

(see in Appendix a remark about collisions).

It is worth pointing out that $A(\Omega)$ depends on $E(t)^{2}$ and not on a product of the electric field at two different times $E\left(t_{1}\right) \cdot E\left(t_{2}\right)$. This arises from the very short time spent by the atom in the relay level, the absorptions of the two photons being practically simultaneous.

The function $A(\Omega)$ involves the relative phases of the various components $\delta(\omega)$ of the field. This can be illustrated by a simple example :

Consider an electromagnetic field which is the superposition of $2 \mathrm{~N}$ monochromatic equidistant modes of frequencies $\omega_{n}$ and amplitudes $E_{n}$. The modes are set out symmetrically on both sides of the atomic resonance, and the distance between modes is large compared with the natural width $\Gamma_{\mathrm{e}}$ of the excited level. A resonance occurs when the atom absorbs a photon from each of the modes symmetric with respect to $\omega_{\mathrm{ge}} / 2$. Replacing integrals by finite sums, the absorbed power is proportional to

$$
A=\left|\sum_{n=1}^{2 N} E_{n} \cdot E_{2 N-n}\right|^{2}=4\left|\sum_{n=1}^{N} E_{n} \cdot E_{2 N-n}\right|^{2} .
$$

We suppose that the amplitudes $E_{n}$ are all equal to $E$ :
If the relative phases are random this leads to

$$
A=4 \sum_{n=1}^{N}\left|E_{n} E_{2 N-n}\right|^{2} \sim 4 N E^{4} .
$$

If all the phases are the same (case of mode-locked laser), there is a constructive interference

$$
A=4\left(N E^{2}\right)^{2}=4 N^{2} E^{4} .
$$

The physical meaning is simple : when the phases of the modes are random, the electric field is almost constant in time. On the contrary, if all the modes have the same phase, $E(t)$ is zero most of the time but very intense over short time intervals. Two-photon absorption being a non-linear process, then the signal is larger in the second case. This effect has been demonstrated in multiphoton ionization [19].

3. Application. The effect of finite transit time. The atoms in a vapour are often moving fast enough for the time spent in the electromagnetic beam to be as short as the atomic lifetime, especially since the laser beam is usually focussed in order to improve the transition probability. This results in an important modification of the line-shape, which we will calculate following the derivation given by Biraben [20].

With the laser operating in a single longitudinal TEM $_{00}$ mode, the spatial distribution of the electromagnetic field can be described by a Gaussian function. The atoms are observed in the vicinity of the waist of the focussed Gaussian beam over a length $L$ which is small compared to the Rayleigh length $z_{\mathrm{R}}[20,22]$. We suppose that the two counter propagating light beams have identical geometry, but may have different intensities. Assuming the same linear polarization for the two beams, then the expression of the electric field is

$$
E(x, y, z, t)=\mathrm{e}^{-\left(x^{2}+y^{2}\right) / w_{0}^{2}}\left[E_{1} \mathrm{e}^{-i\left(\omega_{\mathbf{L}}-k z\right)}+E_{2} \mathrm{e}^{-i\left(\omega_{L} t+k z\right)}\right]+\text { c.c. }
$$

where $w_{0}$ is the beam radius at the waist.

The method consists in first calculating the energy absorbed by an atom with a well defined trajectory which passes through the beam, and then averaging this quantity over all atoms to obtain an expression for the line-shape.

Let us consider an atom following a straight line trajectory whose projection on a plane perpendicular to the beam axis is represented in figure 3 .

Let $\rho$ be the minimum distance between the atomic trajectory and the beam axis, and let $v_{\mathrm{r}}$ and $v_{z}$ denote respectively the radial and axial components of the velocity. We express the atomic coordinates as functions of time (the time origin being when the atom passes at the minimum distance $\rho$ ); we then deduce the electric field seen by the atom :

$$
E(t)=\mathrm{e}^{-\rho^{2} / w_{0}^{2}} \mathrm{e}^{-v t^{2} t^{2} / w_{0}^{2}}\left[E_{1} \mathrm{e}^{-i\left(\omega_{\mathrm{L}}-k v_{z}\right) t}+E_{2} \mathrm{e}^{-i\left(\omega_{L} t+k v_{z}\right) t}\right]+\text { c.c. . }
$$

The averaging calculations will be simplified by the fact that the temporal variation of $E(t)$ is independent of $\rho$.

We must first calculate the square of the electric field. In this calculation we retain only the terms with $\mathrm{e}^{-2 i \omega_{\mathrm{L}} t}$ which are efficient for two-photon absorption :

$$
E(t)^{2}=\mathrm{e}^{-2 \rho^{2} / w_{0}^{2}} \mathrm{e}^{-2 v t^{2} / w_{0}^{2}}\left[2 E_{1} E_{2} \mathrm{e}^{-2 i \omega_{L} t}+E_{1}^{2} \mathrm{e}^{-2 i\left(\omega_{L}-k v_{z}\right) t}+E_{2}^{2} \mathrm{e}^{-2 i\left(\omega_{\mathrm{L}}+k v_{z}\right) t}\right] .
$$


Of the three terms in (41) only the first is independent of the atomic velocity and corresponds to the narrow absorption peak without Doppler broadening; we neglect the other two terms, which represent the smallamplitude, Doppler broadened background (see section 1).

Hence, using (35), the Fourier transform $A(\Omega)$ of $E(t)^{2}$ is given by :

$$
A(\Omega)=\frac{1}{\sqrt{2 \pi}} \frac{w_{0}}{v_{\mathrm{r}}} E_{1} E_{2} \mathrm{e}^{-2 \rho^{2} / w_{0}^{2}} \exp \left[-\frac{\left(\Omega-2 \omega_{\mathrm{L}}\right)^{2}}{8 v_{\mathrm{r}}^{2} / w_{0}^{2}}\right] .
$$

The total energy absorbed by the atom is then (formula (38))

$$
W\left(v_{\mathrm{r}}, \rho\right)=\frac{2 \omega_{\mathrm{L}} w_{0}^{2}}{\hbar v_{\mathrm{r}}^{2}} E_{1}^{2} E_{2}^{2} \mathrm{e}^{-4 \rho^{2} / w_{0}^{2}}\left|\left\langle\mathrm{~g}\left|Q_{\varepsilon \varepsilon}\right| \mathrm{e}\right\rangle\right|^{2} \int_{-\infty}^{+\infty} \mathrm{d} \Omega \frac{\Gamma_{\mathrm{e}}}{\left(\Omega-\omega_{\mathrm{ge}}\right)^{2}+\Gamma_{\mathrm{e}}^{2} / 4} \exp \left[-\frac{\left(\Omega-2 \omega_{\mathrm{L}}\right)^{2}}{4 v_{\mathrm{r}}^{2} / w_{0}^{2}}\right] .
$$
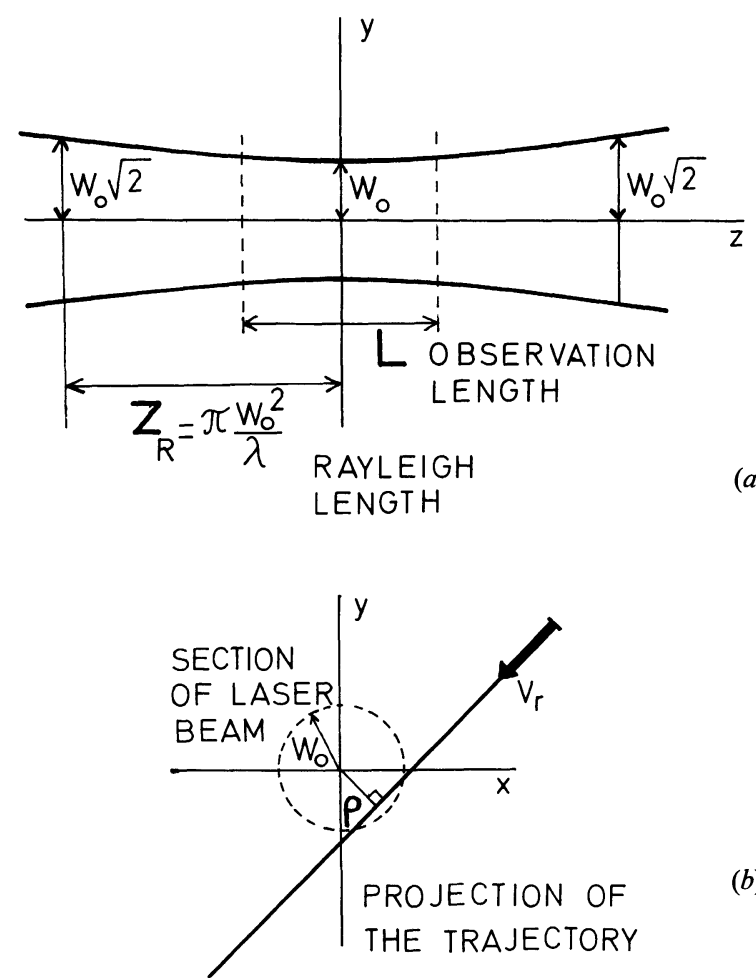

Fig. 3. - The waist of a Laser beam : $a$ ) Longitudinal section showing the observation length $L$, which is supposed to be shorter than the Rayleigh range $z_{\mathrm{R}}$. b) Transverse section, at the waist, showing the projection of an atomic trajectory, with the radial velocity $v_{\mathbf{r}}$.

For a given velocity, the absorption curve is a Voigt profile, convolution of a Lorentzian and a Gaussian curves. It is now necessary to average this expression over the distributions of radial velocities $v_{\mathrm{r}}$ and trajectories (characterized by $\rho$ ), thus obtaining the energy absorbed in unit time. The energy absorbed by all those atoms which pass through the beam in a time $\delta t$ at any point along a length $L$ is

$$
\mathfrak{T} . \delta t=N \int_{0}^{+\infty} 2 \mathrm{~d} \rho \int_{0}^{+\infty} \mathrm{d} v_{\mathrm{r}} \cdot \operatorname{L} v_{\mathrm{r}} \delta t f\left(v_{\mathrm{r}}\right) W\left(v_{\mathrm{r}}, \rho\right)
$$

$N$ is the atomic density and $f\left(v_{\mathrm{r}}\right)$ is the radial velocity distribution, which can be deduced from (16) as

$$
f\left(v_{\mathrm{r}}\right)=\frac{2 v_{\mathrm{r}}}{u^{2}} \mathrm{e}^{-v_{\mathrm{r}}^{2} / u^{2}} \quad \text { with } \quad u^{2}=\frac{2 k T}{M}=\overline{v_{\mathrm{r}}^{2}} .
$$

Replacing $f\left(v_{\mathrm{r}}\right)$ and $W(r, \rho)$ by their expressions, and integrating over $\rho$ and $v_{\mathrm{r}}$ one obtains

$$
\mathfrak{T}\left(\omega_{\mathrm{L}}\right)=C \int_{-\infty}^{+\infty} \frac{\Gamma_{\mathrm{e}}}{\left(\Omega-\omega_{\mathrm{ge}}\right)^{2}+\Gamma_{\mathrm{e}}^{2} / 4} \mathrm{e}^{-\left|\Omega-2 \omega_{\mathrm{L}}\right| / \delta} \mathrm{d} \Omega
$$

where $\delta=u / w_{0}$ and $C$ depends both on atomic parameters and on parameters describing the laser beam

$$
C=N L \pi w_{0}^{2} \frac{\omega_{\mathrm{L}}}{\delta} \frac{E_{1}^{2} E_{2}^{2}}{\hbar}\left|\left\langle\mathrm{g}\left|Q_{\varepsilon \varepsilon}\right| \mathrm{e}\right\rangle\right|^{2}
$$

The line-shape given by (46) is in agreement with that calculated by Bordé [11] in the case of a three level system :

The absorption profile as a function of $\omega_{\mathrm{L}}$ is the convolution of a Lorentzian curve of width $\Gamma_{\mathrm{e}} / 2$ with a double exponential curve of width $\delta \log 2$ at half maximum. The parameter $\delta$ characterizes the broadening due to the transit time through the laser beam. This broadening is symmetrical and the two-photon absorption lines are not shifted by this effect. Let us point out that the particular sharppoint shape of the broadening is due to slow atoms, which stay a long time in the beam and contribute much to be absorption but very little to the broadening. It can also be remarked that the calculation is not valid for atoms of very low radial velocity $v_{\mathrm{r}}$, since such atoms do not have sufficient speed to pass through the beam during the time interval $\delta t$. Nevertheless, it can be shown that the corresponding correction is negligible [20].

In the case where the transit time $1 / \delta$ is much larger than the lifetime of the excited state, i.e. $\delta \ll \Gamma_{\mathrm{e}}$, the broadening associated with the double exponential curve will be negligible. The double exponential 
function in (46) can be replaced by a delta function and the resulting expression for the absorbed power is :

$$
\mathfrak{T}\left(\omega_{\mathbf{L}}\right)=\left(2 \hbar \omega_{\mathbf{L}}\right) \frac{N L \pi w_{0}^{2}}{4} \cdot \Gamma_{\mathbf{g e}}^{(2)}
$$

where $\Gamma_{\mathrm{ge}}^{(2)}$ is the two-photon transition probability in the centre of the light beam, being a Lorentzian function of $\omega_{\mathrm{L}}$, as given by (14). The total power absorbed in the waist of the Gaussian beam is equivalent to that absorbed in the strongest electric field found in a cylinder of radius $w_{0} / 2$. Formula (48) could alternatively be obtained directly by integrating the transition probability (14) over the volume of the Gaussian beam.

4. Experimental profiles. - We present in this section experimental recordings of a particular twophoton absorption line in order to exemplify how the formulas derived above may be applied. The line chosen is the highest frequency component in the set of four which constitute the two-photon absorption by the transition $3 \mathrm{~S} \rightarrow 4 \mathrm{D}$ in sodium, at a wavelength of $5787.3 \AA$, and illustrated in figure 2. The line corresponds to the transition from the hyperfine level $F=2$ of the $3^{2} S_{1 / 2}$ ground state to the finestructure level $4 D_{5 / 2}$. The hyperfine structure of the excited level $4 D_{5 / 2}$ is contained well within the level's natural width of $\Gamma_{\mathrm{e}} / 2 \pi=3.2 \mathrm{MHz}$ and can be completely neglected $[23,24]$.

The experimental set-up is basically that described in earlier publications $[25,26,4]$. However, the feed-back loop which controls the dye laser frequency has been improved and this has allowed frequency jitter to be reduced to less than $1 \mathrm{MHz}$. In order to obtain a linear scan of the laser frequency with time, the external etalon to which the frequency is locked is pressure swept.

The two-photon resonance is monitored by detecting, with a photomultiplier, the photons emitted by the transition between the excited level and the intermediate level $3 \mathrm{P}_{3 / 2}$ : the wavelength $5688 \AA$ of this fluorescence is sufficiently different from that of the laser light for the two to be easily separated by a monochromator. Figures 4 and 5 give two examples of the variation of the photomultiplier current versus time, that is, of the excited state fluorescence as a function of the laser frequency, as drawn with a chart recorder. The horizontal, frequency axis has been scalęd using frequency markers furnished by a long and stable Fabry-Perot interferometer. As is often done in experiments of this kind, the laser beam is focussed into the experimental cell in order to increase the two-photon signal [10]. For example the curve shown in figure 4 was obtained with the laser beam focussed by a lens of focal length $25 \mathrm{~cm}$. The corresponding beam waist radius, $w_{0}$, may be

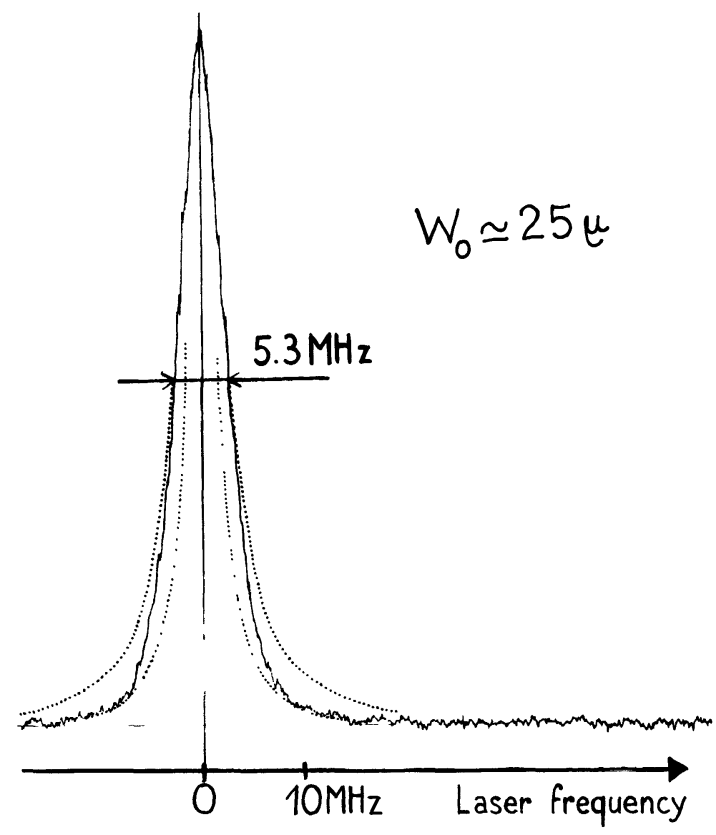

Fig. 4. - Experimental recording with enlarged frequency scale of one component of the two-photon transition $3 \mathrm{~S} \rightarrow 4 \mathrm{D}$ in sodium (component $a$ of figure $2: 3 \mathrm{~S}_{1 / 2} \mathrm{~F}=2 \rightarrow 4 \mathrm{D}_{5 / 2}$ ). The Laser beam is focussed with a lens of focal length $25 \mathrm{~cm}$, giving a waist radius $w_{0} \simeq 25 \mu \mathrm{m}$. The superimposed dotted lines are Lorentzian curves : one coinciding on the wings, the other one coinciding at half-height.

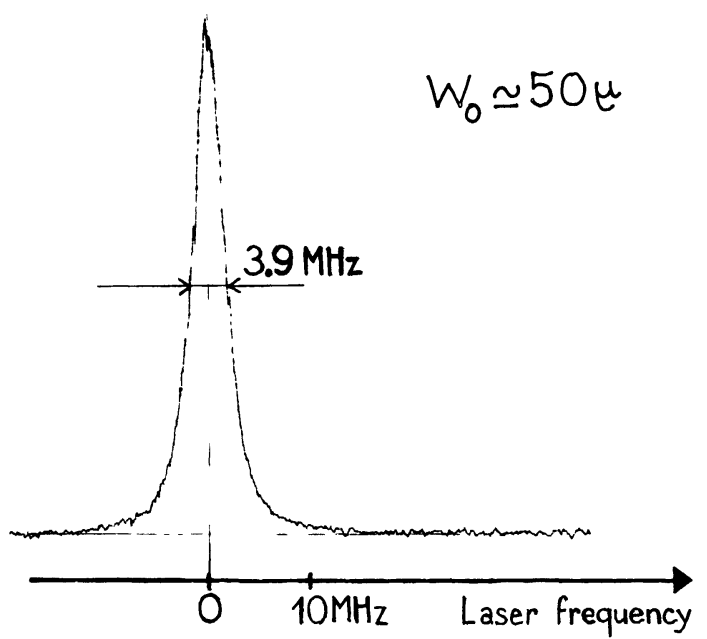

Fig. 5. - Experimental recording of the same transition as figure 4 with a lens of focal length $50 \mathrm{~cm}$ giving a waist radius $w_{0} \simeq 50 \mu \mathrm{m}$, i.e. double that in figure 4 .

estimated to be of the order of $25 \mu \mathrm{m}$, and, with a root-mean-square radial velocity at $180^{\circ} \mathrm{C}$

$$
u=\sqrt{\bar{v}_{\mathrm{r}}^{2}}=570 \mathrm{~ms}^{-1}
$$

(as calculated from (45), we may deduce that the transit time of the atoms through the light beam, given by $w_{0} / u=1 / \delta$, is almost equal to the lifetime of the 4D level, viz. $\left.1 / \Gamma_{\mathrm{e}}=5 \times 10^{-8} \mathrm{~s}[27,28]\right)$. In fact, it is evident from an inspection of figure 4 that, under these conditions, the experimental line-shape 
is quite different from a Lorentzian profile. The two dotted curves superimposed on the experimental curve of figure 4 are two Lorentzians : the first curve coinciding at the wings with the experimental curve; the second having the same width at half-height as the observed line-shape. The rapid decrease in the signal in the wings is characteristic of the double exponential curve which we have introduced in the above analysis of transit time broadening (46).

In order to measure more precisely the effect of the transit time, another recording was made of the two-photon signal with the laser beam focussed with a lens of focal length $50 \mathrm{~cm}$, thus producing a beam waist twice as large (i.e. $w_{0} \simeq 50 \mu \mathrm{m}$ ) as that associated with the above recording. The corresponding curve, shown in figure 5, is narrower and much closer to the Lorentzian shape.

When out the convolution of a Lorentzian curve with a double exponential curve (the widths of which are of the same order of magnitude) is carried out then numerical calculation shows that the width of the resulting curve is nearly the sum of the widths of the two convoluted curves. This property permits an easy comparison of the two experimental lineshapes shown in figures 4 and 5 .

We associate the form of each experimental line-shape with that given by the theoretical expression (46); the two curves having different values, $\delta_{4}$ and $\delta_{5}$, of the transit time parameter. Knowing that $\delta_{4}=2 \delta_{5}$, we can write the differences of the widths as

$$
\delta_{5} \frac{\log 2}{2 \pi}=\frac{1}{2} \delta_{4} \frac{\log 2}{2 \pi}=1.4 \mathrm{MHz}
$$

and thus deduce that

$$
\delta_{4}=2 \delta_{5}=2.5 \times 10^{7} \mathrm{~s}^{-1} .
$$

This value is in good agreement with the value deduced from the estimated waist radius $w_{0}$ of the beam and the r.m.s. radial velocity of the atoms. In explaining the widths of the experimental curves in figures 4 and 5 , we know that the contribution of the radiative lifetime is $\Gamma_{\mathrm{e}} / 4 \pi=1.6 \mathrm{MHz}$, being the full width at half-maximum of the Lorentzian curve. In addition, however, it is necessary to include an additional contribution of $1 \mathrm{MHz}$, which arises in part from the residual frequency jitter of the laser, and in part from the collisional broadening (the latter due to the presence of impurities in the experimental cell which broaden the Lorentzian curve).

In conclusion, we have established a general formalism which permits the calculation of lineshapes in Doppler-free, two-photon spectroscopy. We have applied this general formalism to include the effects of finite transit times of the atoms through the laser beam, and we have found good agreement between theoretical and experimental line-shapes.

We wish to thank Gilbert Grynberg and Elisa- beth Giacobino for many valuable discussions on the topic of this paper, and Leslie Pendrill for assistance with the language.

Appendix. - Collisional efFeCts ON TWO-PHOTON LINE-SHAPES. - Much detailed work has been performed on the effects of collisions on spectral line-shapes. Here we wish to concern ourselves with only one aspect ; the quite different effects of collisions on Doppler-free, two-photon line-shapes on the one hand, and on saturated absorption line-shapes, on the other.

We emphasised in section 2 that the two-photon line-shape depends, through the quantity $A(\Omega)$, on $E^{2}(t)$ and not on a product of the electric field at two different times : see formulas (35) and (36). This remark also has some relevance in the analysis of the two-photon line-shape in the presence of collisions which modify the atomic velocity. The result is that the two-photon line-shape is independent of the atomic trajectory, that is, insensitive to velocitychanging collisions, in contrast to the pronounced effects of collisions on saturated absorption profiles.

The atomic trajectory is given by the vector $\mathbf{r}(t)$. At a given time $t$, the atom interacts with an electric field (as experienced in the atomic rest frame)

$$
\mathbf{E}(t)=\varepsilon\left[E_{0} \mathrm{e}^{-i \omega_{\mathbf{L}} t-i \mathbf{k} . \mathbf{r}(t)}+E_{0} \mathrm{e}^{-i \omega_{\mathbf{L}} t+i \mathbf{k} . \mathbf{r}(t)}\right]
$$

in the case of a monochromatic standing wave.

Deducing the corresponding expression for $A(\Omega)$, the Fourier transform of $E^{2}(t)$, from equation (35), we see that it consists of three terms, one of which is independent of the trajectory, i.e. of $\mathbf{r}(t)$. As mentioned in the text, this term gives the form of the Doppler-free, two-photon line. It follows that the two-photon absorption line-shape is not sensitive to velocity-changing collisions.

The same range of validity applies for this result as with the analysis leading to formula (36) in the text. In particular, one must exclude single-photon absorption : the energy defect, $\hbar \Delta \omega_{\mathrm{r}}$, has to be large compared with the spectral width of the electromagnetic field as it appears in the atomic frame. The spectral width in this case is of the order of $1 / \tau_{c}$, where changes in the apparent frequency of the light field occur (due to changes in velocity of the atom) in times of the order of $\tau_{c}$, the collision time.

We can then neglect velocity-changing collisions provided

$$
\Delta \omega_{\mathrm{r}} \gg 1 / \tau_{\mathrm{c}} .
$$

In other words, the time taken for the two-photon absorption process to occur $-1 / \Delta \omega_{\mathrm{r}}-$ must be short compared to the collision time, $\tau_{\mathrm{c}}$. Such a condition excludes the case where a collision takes place between the absorption of two photons.

In conclusion, the effects of collisions on twophoton absorption result in a Lorentzian line-shape, and can thus be described by two quantities : the shift of the line's centre and the broadening. 


\section{References}

[1] Biraben, F., Cagnac, B. et Grynberg, G., Phys. Rev. Lett. 32 (1974) 643.

[2] Levenson, M. D. and Blombergen, N., Phys. Rev. Lett. 32 (1974) 645.

[3] Hansch, T. W., Harvey, K., Meisel, G. and Schawlow, A. L., Opt. Commun. 11 (1974) 50.

[4] Grynberg, G. and Cagnac, B., Rep. Prog. Phys. 4 (1977) 791.

[5] Borde, C. J., Hall, J. L., KunASz, C. V. and Hummer, D. G., Phys. Rev. A 14 (1976) 236.

[6] Thomas, J. E., Kelly, M. J., Monchalin, J. P., Kurnit, N. A. and JAVAN, A., Phys. Rev. A 15 (1977) 2356.

[7] Rautian, S. G. and Shalagin, A. M., Sov. Phys. JetP 31 (1970) 518.

[8] Baklanov, E. V., Dubetski, B. Y., Semibalamut, V. M. and Titov, E. A., Sov. J. Quant. Electron. 5 (1976) 1374.

[9] Vasilenko, L. S., Chebotaev, V. P. and Shishaev, A. V., J.E.T.P. Lett. 11 (1970) 113.

[10] Cagnac, B., Gynberg, G. and Biraben, F., J. Physique 34 (1973) 845.

[11] Borde, C., C.R. Hebd. Séan. Acad. Sci. 282 (1976) B 341.

[12] Grynberg, G., Thesis Paris 1976 - unpublished CNRS AO 12497.

[13] Flusberg, A., Mossberg, T. and Hartman, S. R., Phys. Rev. A 14 (1976) 2146.

[14] Grynberg, G., Biraben, F., Giacobino, E. and Cagnac, B., J. Physique 38 (1977) 629.
[15] Cohen-Tannoudi, C., Cargèse Lectures on Physics (Gordon and Breach) 1967.

[16] Heitler, W., The Quantum Theory of Radiation (Clarendon Press) 1954.

[17] Biraben, F., Cagnac, B. and Grynberg, G., C.R. Hebd. Séan. Acad. Sci. 279 (1974) B 51.

[18] Anderson, P. W., Phys. Rev. 76 (1949) 647 ; see also SOBEL'MAN « An Introduction to the Theory of Atomic Spectra » (Pergamon) 1969.

[19] Lecompte, G., Mainfray, G., Manus, C. and Sanchez, F., Phys. Rev. Lett. 32 (1974) 265.

[20] Biraben, F., Thesis Paris 1977 - unpublished.

[21] Kogelnik, H. and LI, T., Appl. Opt. 5 (1966) 1550.

[22] Siegman, A. E., An Introduction to Lasers and Masers (Mac Graw Hill) 1971.

[23] Biraben, F. and Beroff, K., Phys. Lett. 65A (1978) 209.

[24] Burghart, B., Dubke, M., Jitschin, W. and Meisel, G., Communication to 10th EGAS Conference Münich (1978).

[25] Biraben, F., Giacobino, E. and Grynberg, G., Phys. Rev. A 12 (1975) 2444.

[26] Giacobino, E., Biraben, F., Grynberg, G. and Cagnac, B., J. Physique 38 (1977) 623.

[27] Karsten, F., Schramm, J., Z. Phys. 195 (1970) 360.

[28] KAISER, D., Phys. Lett. 53A (1975) 61. 